\title{
Recursive least square and fuzzy modelling using genetic algorithm for process control application
}

\begin{abstract}
A technique for the modelling of nonlinear process control using Recursive Least Square and Takagi-Sugeno Fuzzy System with Genetic Algorithm topology is described. This paper discusses the identification of parameters of the fuzzy sets at the antecedent part and linear model at the consequent part of fuzzy model within an application to process control. The key issues of finding the best model of the process are described. Results show that fuzzy model with genetic algorithm gives minimum mean squared error compare with recursive least square.
\end{abstract}

Keyword: Recursive least square; Process control; Nonlinear process control; Fuzzy modelling 